\title{
ORIGINAL
}

\section{BROTE DE ESCABIOSIS EN UNA RESIDENCIA PARA PERSONAS MAYORES EN LA PROVINCIA DE HUESCA}

\author{
Alberto Larrosa Montañés (1), Manuel Cortés Blanco (1,2), Carlos Clerencia Casorrán (3), Silvia \\ Martínez Cuenca (1), Javier Urdániz Sancho (3), Julián Urbán Sender (3), Cristina Ariño Alda- \\ bo (4) y Luis Güerri Mir (4) \\ (1) Sección Regional de Vigilancia Epidemiológica. Servicio de Prevención y Promoción de la Salud. Servicio Ara- \\ gonés de la Salud. Zaragoza. \\ (2) Programa Epidemiología Aplicada de Campo. Centro Nacional de Epidemiología. Instituto de Salud Carlos III. \\ Madrid. \\ (3) Sección Provincial de Vigilancia Epidemiológica. Servicio de Prevención y Promoción de la Salud. Servicio Ara- \\ gonés de la Salud. Huesca. \\ (4) Centro de Atención Primaria de Barbastro. Barbastro (Huesca).
}

\section{RESUMEN}

Fundamento: Los colectivos de personas institucionalizadas constituyen actualmente un grupo de riesgo para la aparición de brotes de escabiosis. El objetivo de este trabajo es estudiar desde la Sección Provincial de Vigilancia Epidemiológica de Huesca las características de uno de esos brotes, ocurrido en una residencia para personas mayores ubicada en el municipio de Barbastro (Huesca).

Métodos: Tras la notificación inicial de casos, se estableció un protocolo específico para la prevención de escabiosis. Se llevó a cabo una investigación epidemiológica, procediéndose a la búsqueda activa de casos entre todos las personas residentes y los trabajadores. La información obtenida de los casos y contactos mediante una encuesta validada fue incluida en una base de datos utilizando Epiinfo 6.0. Se estimó la tasa de ataque y el riesgo relativo de los residentes de cada área del centro, tomando como referencia la de menor tasa de ataque.

Resultados: Entre las 104 personas residentes y 20 trabajadores, se detectaron 19 casos de escabiosis (18 y 1 respectivamente). No pudo precisarse el origen del brote, si bien al menos dos de los primeros casos iniciaron sus síntomas seis meses antes de su detección. El retraso diagnóstico medio fue de 53 días. El brote afectó preferentemente a la sección de hombres asistidos $\left(\mathrm{TA}=77 \% ; \mathrm{RR}=18,5, \mathrm{IC}_{05 \%}=2,7-128,7\right)$. El único trabajador afectado era el responsable de este área, quien no aplicaba durante sus cuidados las medidas universales de protección individual.

Conclusiones: Se trata de un brote de escabiosis en una residencia para personas mayores, probablemente debido al retraso diagnóstico de los primeros casos y a la falta de uso de las medidas de protección indi por parte de un cuidador. La aplicación de un protocolo específico para la prevención de escabiosis, resultó fundamental para su control.

Palabras clave: Escabiosis. Infestaciones por ácaros. Brote epidémico. Hogares para ancianos.

Correspondencia:

Alberto Larrosa Montañés.

Servicio Aragonés de la Salud.

Servicio de Prevención y Promoción de la Salud.

Sección Regional de Vigilancia Epidemiológica.

Ramón y Cajal 68.

50.004 Zaragoza

Correo electrónico: alarrosa@aragob.es

\section{ABSTRACT}

Scabies Outbreak at a Senior Citizen Living Facility in the Province of Huesca, Spain

Background: Individuals residing in institutionalized living facilities are currently a high-risk group as regards scabies outbreaks. This study is aimed at analyzing the characteristics of one of these outbreaks, which occurred at a senior citizen living facility located in the municipality of Barbastro (Huesca) through the Huesca Provincial Epidemiologi cal Monitoring Division.

Methods: Following the initial notification of cases, a specific scabies prevention protocol was set out. An epidemiological investigation was conducted proceeding to an active search for cases among all of the indiiduas resing or woling at the facility in question The if th an contion gathere on the cases and contacts by means of a valinded survey was included in a database using Epinfo 6.0. The attack rate and the relative risk of those residing in each area of the center were estimated, taking as a reference that having the lowest attack rate.

Results: A total of nineteen (18 and 1 respectively) cases of scabies were detected among the 104 residents and 20 workers employed at the facility. The source of the outbreak could not be specifically determined, although at least two of the first cases had begun to show some symptoms six months prior to their detection. The average delay in diagnosis was 53 days. This outbreak had a greater effect on the males in the assisted living quarters $(\mathrm{TA}=77 \%$; $\mathrm{RR}=18.5, \mathrm{Cl} 95 \%=2.7-128.7)$. The only employee affected was the person in charge of this area, who had not employed the universal personal protection measures during his caregiving tasks.

Conclusions: This is a scabies outbreak at a senior citizen living facility, probably due to the delay in diagnosing the first cases and the failure on the part of one caregiver to have employed the personal protection measures. A specific scabies-prevention protocol having been implemented was fundamental for the control of this outbreak.

Key words: Scabies. Mites. Epidemic outbreak. Homes for the aged. 


\section{INTRODUCCIÓN}

Las residencias para personas mayores pueden constituir centros de riesgo para la presentación de brotes de escabiosis o sarna ${ }^{1}$. Entre los ancianos es habitual que el cuadro clínico de esta enfermedad sea inespecífico, que no presenten reacciones por hipersensibilidad y que asocien el padecimiento de otras patologías concomitantes. Todo ello dificultaría su diagnóstico, prolongando la exposición inadvertida de sus contactos (incluyendo sus propios cuidadores) y pudiendo generar brotes a nivel de la institución ${ }^{2,3}$.

Por otra parte, la afectación de algún caso entre el personal laboral de estos centros suele reflejar la existencia de cargas de trabajo inadecuadas, falta de información al respecto (como el desconocimiento de los mecanismos de transmisión) y/o defectos en la aplicación de las debidas medidas preventivas $^{4}$.

El objetivo de este trabajo es describir las características de un brote de escabiosis ocurrido en una residencia para personas mayores, destacando la importancia que tuvo para su control y extinción el establecimiento precoz de las oportunas medidas preventivas.

\section{SUJETOS Y MÉTODO}

El día 29/11/2002 el Centro de Atención Primaria de Barbastro (Barbastro, Huesca) notificó a la Sección Provincial de Vigilancia Epidemiológica de Huesca la existencia de seis casos de escabiosis en una residencia para personas mayores de dicho municipio.

Desde dicha Sección Provincial, y en colaboración con el coordinador de ese Centro de Atención Primaria, el día 3/12/2002 se procedió a la inspección de la residencia y a la búsqueda activa de casos de escabiosis entre sus residentes y trabajadores.
La institución cuenta con 20 trabajadores habituales y 104 residentes divididos en cuatro áreas independientes: 29 hombres válidos (área 1), 13 hombres asistidos (área 2), 38 mujeres válidas (área 3) y 24 mujeres asistidas (área 4). No obstante, dispone de diferentes espacios comunes (comedores, salas de reunión, peluquería, etc.) en los que los residentes se juntan y desarrollan sus actividades cotidianas.

Para la búsqueda de casos se realizó la exploración corporal de todos sus residentes y trabajadores, y una entrevista personal utilizando una encuesta epidemiológica previamente validada desde la Sección Regional de Vigilancia Epidemiológica del Servicio Aragonés de la Salud (Anexo 1). En aquellas personas a las que no pudo entrevistarse (debido fundamentalmente a patologías concomitantes o dificultades en el proceso de comunicación), se procedió a la revisión de sus historias clínicas y a la obtención de datos a través de sus cuidadores o familiares.

En el estudio se asumieron las siguientes definiciones:

- Caso sospechoso. Residente o trabajador del referido centro que hubiera presentado o tuviese documentados durante las últimas seis semanas (máximo período de incubación para escabiosis): Prurito de varios días de evolución, fuera generalizado o localizado y aparición de lesiones cutáneas sugestivas de escabiosis, con independencia de su extensión y gravedad. También se consideraron casos sospechosos a los contactos de dichos casos que presentasen estos mismos signos y síntomas.

- Caso confirmado. Residente o trabajador del centro, o contactos de cualquiera de ellos, en quienes se comprobase la existencia de Sarcoptes scabiei por visión directa, anatomía patológica u otros métodos de análisis microbiológico. En nuestro estudio, la confirmación 
se estableció mediante visión directa del ácaro en piel por el servicio de dermatología del Hospital Comarcal de Barbastro.

- Contacto: Persona que cumpliese alguno de los siguientes criterios:

a. pertenecer a la plantilla de trabajadores de la residencia.

b. ser residente de dicho centro.

c. haber tenido un contacto físico estrecho en las últimas seis semanas (máximo período de incubación para escabiosis) con alguno de los casos. Se trataba fundamentalmente de familiares y amigos que iban a visitarlos a la institución. Estos contactos fueron localizados desde el Centro de Atención Primaria de Barbastro, siendo valorados y encuestados por su equipo médico.

- Retraso diagnóstico. Diferencia de tiempo entre la fecha de inicio de los síntomas de escabiosis y la fecha de su diagnóstico médico.

Entre los trabajadores de la residencia se analizó su aplicación de las medidas preventivas de protección universal durante el desarrollo de sus cuidados, a través de la encuesta epidemiológica y la inspección directa de los mismos.

La información obtenida sobre casos y contactos fue incluida en una base de datos utilizando el programa Epiinfo 6.0. Se estimó el riesgo relativo de los residentes de cada una de las áreas de la institución, tomando como referencia la de menor tasa de ataque; asimismo, se establecieron sus respectivos intervalos de confianza con un nivel de significación del $95 \%(\alpha=0,05)$.

Atendiendo a las características del centro y la propia enfermedad, el día 5/12/2003 se aplicó tratamiento simultáneo con permetri- na crema al 5\% a todo su personal (casos y no casos, residentes y trabajadores por igual), así como al resto de los contactos que pudieron establecerse (en total 17 familiares de los casos). La aplicación fue por todo el cuerpo, excepto en la cara y plantas de los pies, prestándose especial atención a los espacios interdigitales. Tras dejarla actuar 24 horas, se recomendó una ducha integral y el cambio de las ropas personales y de cama, procediéndose simultáneamente a la limpieza exhaustiva de todo el establecimiento ${ }^{5,6}$. Durante esas 24 horas se restringió el contacto con otras personas a todos los casos detectados y se indicó a la cuidadora infestada que no realizara sus tareas habituales. Las ropas retiradas se introdujeron en bolsas de plástico cerradas y se mantuvieron en el exterior durante 4-5 días (el parásito no vive más de 24-48 horas fuera del cuerpo humano y es muy sensible a la desecación y las temperaturas extremas) $)^{6}$. Posteriormente fueron lavadas a máquina en ciclos calientes.

Todos los casos fueron revisados dos semanas después, recomendándose una nueva aplicación a aquellos en que se considerara necesario. A las seis semanas se hizo otra revisión (abarcando el máximo período de incubación de escabiosis) para evaluar la efectividad de las medidas aplicadas. Todas estas revisiones fueron realizadas por los mismos facultativos que diagnosticaron los casos iniciales, manteniéndose así criterios semejantes en su valoración.

\section{RESULTADOS}

Se detectaron un total de 19 casos sospechosos de escabiosis entre trabajadores (1) y residentes (18) de la institución. Dos de ellos fueron confirmados mediante visión directa del ácaro en sus lesiones cutáneas.

El caso índice fue diagnosticado el día 19/11/2002 por su médico de cabecera.

De los 18 casos residentes, nueve pertenecían al área de hombres asistidos (tasa de 
Tabla 1

Riesgo relativo e intervalos de confianza de padecimiento de escabiosis para cada área de la residencia con relación a la de menor tasa de ataque

\begin{tabular}{|c|c|c|}
\hline Área & Riesgo relativo & Intervalo Confianza $\left(\mathbf{I C}_{\mathbf{9 5}} \mathbf{\text { }}\right)$ \\
\hline Área 1 (hombres válidos) & 3,3 & $0,4-27,7$ \\
\hline Área 2 (hombres asistidos) & 18,5 & $2,7-128,7$ \\
\hline Área 3 (mujeres válidas) & 1,9 & $0,2-17,2$ \\
\hline Área 4 (mujeres asistidas) & 1 & - Referencia- \\
\hline
\end{tabular}

ataque: $77 \%$ ), cinco a la de hombres válidos $(14 \%)$, uno a la de mujeres asistidas $(4 \%)$ y tres a la de mujeres válidas $(8 \%)$. El caso aparecido entre los trabajadores era una persona que vivía en la propia institución y era responsable principal de los cuidados del área de hombres asistidos.

Entre el resto de trabajadores y los 17 contactos valorados fuera del centro no se detectó ningún caso secundario.

En la tabla 1, se presentan los riesgos relativos para cada una de las áreas con relación a la de menor tasa de ataque (área de mujeres asistidas).

La edad media de los casos fue de 75,2 años (rango: 58-92), con una mediana de 76. Sus síntomas y signos se distribuyen de la siguiente forma: $18(95 \%)$ prurito (siendo evidentes las lesiones secundarias de rascado), 15 (79\%) pápulas, $12(63 \%)$ eritema y 8 (42\%) vesículas. No se constatan otros síntomas generales. Estas lesiones se localizaban preferentemente en brazos y antebrazos (74\%, incluyendo pliegues axilares), tronco (58\%), pies y piernas (37\%), y región inguinal $(32 \%)$.

El retraso diagnóstico medio fue de 53 días (rango: 7-180), con una mediana de 60. Al menos hubo dos casos sospechosos que iniciaron sus síntomas seis meses antes de su diagnóstico. Se trataba de dos hombres mayores (81 y 92 años de edad), postrados permanentemente en cama, con patología crónica concomitante (ambos padecían alteraciones en la glucemia y uno hipertensión arterial), un notable deterioro psíquico (con las consecuentes dificultades para comunicarse) y un alto grado de dependencia física, pertenecientes al área 2 (hombres asistidos). Presentaban lesiones eritematosas y descamativas generalizadas, localizadas preferentemente en espalda y extremidades, que en ambos casos fueron reconocidas en una ocasión por su médico de cabecera. El segundo de ellos, coincidiendo con un ingreso hospitalario, fue también valorado por un dermatólogo. Fueron siempre diagnosticados de «dermatitis inespecífica», prescribiéndose tratamiento sintomático (antihistamínicos orales y corticoides tópicos) que no produjo la curación.

De entre los seis casos declarados en un inicio y los 13 más detectados a través de la búsqueda activa no se constataron diferencias significativas (ni siquiera en el retraso diagnóstico), posiblemente debido al reducido tamaño muestral. La única diferencia relevante fue que el cuadro dermatológico de los seis casos declarados había sido valorado al menos una vez por sus respectivos médicos de cabecera en los seis meses previos, frente a sólo dos de los encontrados por búsqueda activa. En ninguno de ellos se estableció inicialmente el diagnóstico de escabiosis, recibiendo tratamiento sintomático. 
No se constata relación de este brote con contactos con animales ni con el mantenimiento de relaciones íntimas con otras personas.

Todos los cuidadores de la institución, excepto uno (precisamente el único caso habido entre ellos), aplica con asiduidad las medidas universales de protección individual durante los cuidados de los residentes.

La evolución de las lesiones en los casos con el tratamiento indicado fue buena. No obstante, en 14 de ellos se recomendó una segunda aplicación a las dos semanas a fin de garantizar su completa resolución. No se constataron efectos secundarios de interés. En la revisión a las seis semanas se evidenció la efectividad de las medidas aplicadas, no detectándose ningún caso y considerándose el brote extinguido.

\section{DISCUSIÓN}

Puede ser habitual que en personas mayores y/o inmunodeprimidas afectadas de escabiosis el diagnóstico se retarde debido a la inespecificidad de sus lesiones. De hecho, muchas de ellas son similares a otros procesos cutáneos como eczemas y toxodermias, o a la administración de tratamientos sintomáticos ${ }^{7,8}$. Por otra parte, en dichos colectivos la expresión de la enfermedad puede diferir de su patrón típico, mostrándose con frecuencia como una dermatitis generaliza$\mathrm{da}^{9}$.

Tales circunstancias parecen estar detrás del retraso diagnóstico en el brote que nos ocupa. De ahí la importancia de considerar la posibilidad de una escabiosis en personas mayores y/o inmunodeprimidas que presenten lesiones cutáneas de larga evolución, que no respondan favorablemente al tratamiento instaurado.

Dicho retraso diagnóstico en pacientes potencialmente infestantes, y con una mayor prevalencia de enfermedades concomitantes, puede ocasionar exposiciones prolongadas al ácaro de terceras personas, como sus cuidadores. En este sentido, un único contacto físico entre uno de estos profesionales y un paciente parasitado podría ocasionar su transmisión y la aparición de brotes institucionales $^{8,10,11}$.

En este brote, la investigación epidemiológica no ha permitido precisar el origen del mismo. No obstante, de ella se desprende que la situación se mantuvo en el centro durante al menos seis meses sin un diagnóstico específico, lo que pudo permitir la transmisión por contacto persona a persona. Dicha circunstancia es totalmente distinta a la habida en otros brotes concentrados en el tiempo (y, por tanto, con un menor período de exposición), en los que el origen del mismo está en una fuente común ${ }^{12}$.

Los primeros casos aparecieron en el área de hombres asistidos. Era además la que tenía una mayor tasa de ataque, estando a su cargo el único trabajador infestado del centro.

Este trabajador no utilizaba con asiduidad las medidas universales de protección individual (limpieza exhaustiva de manos antes y después de cada atención, uso de guantes desechables durante la misma, etc.) en el manejo de los residentes de su área. Esto pudo favorecer tanto que él mismo se infestara como que fuese vehículo de transmisión de la enfermedad, al igual que se ha constatado en otros brotes similares ${ }^{4}$. El resto de los trabajadores aplicaba asiduamente en sus cuidados dichas medidas universales.

La presencia de casos en otras áreas podría deberse a los contactos entre residentes en los espacios comunes de la institución o a su relación personal (no tanto por sus cuidados, dada la delimitación de tareas) con el referido cuidador.

Una de las principales dificultades de este estudio fue la realización de las encuestas 
epidemiológicas. Debido al deterioro mental de algunos de los residentes, no fue posible la cumplimentación directa de muchos de sus ítems, procediéndose en ellos a la revisión de sus historias clínicas o incluso a la obtención de datos a través de sus cuidadores o familiares.

El diseño y aplicación de un protocolo específico de escabiosis 13, incluyendo el tratamiento simultáneo y reglado de todos los casos y contactos, la activación de la vigilancia epidemiológica para la detección precoz de nuevos casos y la insistencia a todo el personal laboral de la importancia de mantener durante su labor las medidas universales de protección individual, resultó fundamental para el control del brote.

\section{BIBLIOGRAFÍA}

1. Reintjes R, Oostendorp JJHM, Carsauw HHC, van der Lans SMGA, Conynvan Spaendonck MAE. Investigation of recurring scabies outbreaks in a nursing home [citado 2 de diciembre 1. 2002]. Disponible en: www.epiet.org/seminar/1997/reintjes3.html

2. Voss A, Wallranch C. Occupational scabies in healthcare workers. Infect Control Hosp Epidemiol 1993; 12, 1: 26-33

3. Hernández Cano N, López de Ayala Casado E. Prurito senil. Rev Esp Geriatr Gerontol 2002; 37, 55: 9-18.

4. Gil Alcamí J, Aparici Cruz D, Salazar Cifre A, Roig Sena F, Chofre Navarrete C. Brote de sarna en personal sanitario. Enfermería Integral 1999; 48.
5. Elston DM. Controversies concerning the treatment of lice and scabies. J Am Acad Dermatol 2002; 46: 794-6.

6. Consejería de Sanidad. Protocolo de actuación frente a la sarna. Madrid: Comunidad de Madrid, 2000 .

7. Estes SA, Estes J. Therapy of scabies: nursing homes, hospitals and the homeless. Semin Dermatol 1993; 12, 1: 26-33.

8. Robles García M, de la Lama López-Areal J, Avellaneda Martínez C, Giménez García R, Cortejoso Gonzalo B, Vaquero Puerta JL. Brote nosocomial de escabiosis. Rev Clin Esp 2000; 200: 538-42.

9. Chin J. Scabies. En: Chin J (editor).Control of communicable diseases manual. Washington 2000; $17^{\mathrm{a}}$ edic.: 445-7.

10. Cárdenes Santana MA, Suárez Ortega S, Jiménez Santana P, Carretero Hernández G, Artiles Vicaíno J, Melado Sánchez P. Brote epidémico de escabiosis en relación con un paciente con infección por el virus de la inmunodeficiencia humana y sarna noruega. Rev Clín Esp 1993; 193: $155-8$.

11. Ortega L, Sierra M, Cid R, Rodríguez T, Aparicio M. Epidemiología de un brote nosocomial de sarna. Enferm Infecc Microbiol Clin 1995; 13: 495-6.

12. Guimbao Bescós J, Moreno Marín P, Pac Sa R, Gutiérrez Colás V. Escabiosis en la escuela: descripción de un brote atípico. Med Clin (Barc) 1990; 94: 759.

13. Goins M. Scabies outbreak controlled [citado $18 \mathrm{de}$ diciembre 2002]. Disponible en: www.shmc. $\mathrm{org} / \mathrm{shmcmedialink/pdf/scabies \_ update.pdf}$ 


\section{Anexo 1}

Encuesta epidemiológica de escabiosis utilizada en el estudio de este brote

\section{Datos de filiación}

Apellidos y Nombre:.

Dirección:

Localidad:

Teléfono:

Sexo: Mujer/Hombre

Edad en años. Fecha de Nacimiento:

Estado civil: Soltero/ Casado/ Separado/ Divorciado/ Viudo

Profesión: Personal sanitario/ Personal no sanitario.

Lugar de trabajo (especificar):

Número de miembros de su unidad familiar: Especificar:

\section{Datos clínicos:}

Fecha de inicio de los primeros síntomas y signos:

Fecha del diagnóstico:

Síntomas y signos: Picor/prurito: Generalizado/Localizado/De predominio nocturno

Lesiones cutáneas: Pápulas/Eritema/ Vesículas/Otras

Localización principal: Manos/ Piernas/Genitales / Cara / Pies / Brazos /Tronco/Otras

Otros síntomas:

Caso: Sospechoso/Confirmado/No caso

De ser un caso confirmado, ¿técnica utilizada para la confirmación?:

Visión directa/Microbiología/ Anatomía Patológica/Otras

¿Hay algún otro miembro de la familia con síntomas similares?: Sí/ No/ NS/NC

De ser afirmativa, ¿de quién se trata?

¿Y amigos, vecinos, conocidos, etc.? No/ Sí/ ¿Quién?

¿Ha acudido al médico por estos síntomas?: No/Dermatólogo/Atención primaria/Otros

¿Ha recibido tratamiento?: $\mathrm{No}$ / Sí/Especificar

Fecha de inicio del tratamiento: 
¿Ha padecido alguna reacción adversa al tratamiento?: No/ Sí/Especificar

¿Padece alguna otra enfermedad?: No/ Sí/Especificar

\section{Datos epidemiológicos:}

Especificando en cada respuesta afirmativa las características del contacto

Antes de la aparición de los síntomas y/o signos, ¿ha tenido contacto:

- con otras personas que han padecido o padecen escabiosis (o un cuadro clínico compatible)?

- con ropa de enfermos?

- con animales?

- íntimo con otra persona que no sea su pareja habitual?

Fecha(s) del contacto(s):

Para trabajadores sanitarios:

¿Utilizó medidas de protección individual en dicho contacto?

¿Utiliza medidas de protección individual durante el cuidado de sus pacientes?

Siempre / Casi siempre / A veces /Nunca

¿Qué tipo de medidas de protección?

\section{Observaciones:}

